\title{
Structural abnormalities in gyri of the prefrontal cortex in individuals with schizophrenia and their unaffected siblings
}

Michael P. Harms, Lei Wang, Carolina Campanella, Kristina Aldridge, Amanda J. Moffitt, John Kuelper, J. Tilak Ratnanather, Michael I. Miller, Deanna M. Barch and John G. Csernansky

\section{Background}

The relatives of individuals with schizophrenia exhibit deficits of overall frontal lobe volume, consistent with a genetic contribution to these deficits.

\section{Aims}

To quantify the structure of gyral-defined subregions of prefrontal cortex in individuals with schizophrenia and their siblings.

\section{Method}

Grey matter volume, cortical thickness, and surface area of the superior, middle and inferior frontal gyri were measured in participants with schizophrenia and their unaffected (non-psychotic) siblings ( $n=26$ pairs), and controls and their siblings ( $n=40$ pairs).

\section{Results}

Grey matter volume was reduced in the middle and inferior frontal gyri of individuals with schizophrenia, relative to controls. However, only inferior frontal gyrus volume was also reduced in the unaffected siblings of those with schizophrenia, yielding a volume intermediate between their affected siblings and controls.

\section{Conclusions}

The structure of subregions of the prefrontal cortex may be differentially influenced by genetic factors in schizophrenia, with inferior frontal gyrus volume being most related to familial risk.

\section{Declaration of interest}

None.
The frontal cortex is involved in the processing of a variety of cognitive and behavioural domains that are relevant to the pathophysiology of schizophrenia, including executive function, working memory, language production, attention, motivation and emotional processing. ${ }^{1}$ Studies that have partitioned the frontal cortex according to intrinsic sulcal/gyral anatomy have generally identified the inferior and/or middle frontal gyrus as regions of grey matter loss in individuals with schizophrenia ${ }^{2-6}$ (but see Crespo-Facorro et al). ${ }^{7}$ Studies of the unaffected (nonpsychotic) siblings of individuals with schizophrenia can help to determine whether such findings are due to genetic factors, since siblings share on average $50 \%$ of their genetic variants. Prior studies suggest that the relatives of individuals with schizophrenia exhibit abnormalities of overall frontal lobe volume ${ }^{8,9}$ (but see Staal et al). ${ }^{10}$ However, such studies have not addressed whether gross volume deficits were localised to particular frontal gyri or subregions, which is important because of the specific roles such regions play in cognition. In the present study, we examined the structure of three prefrontal cortical gyri using modern computational neuroanatomy in conjunction with manual gyral delineation in individuals with schizophrenia and their siblings, and in control individuals and their siblings.

\section{Method}

\section{Participants}

The participants included in this study were drawn from a population of individuals who volunteered for studies of brain structure and function at the Conte Center for the Neuroscience of Mental Disorders at Washington University School of Medicine in St Louis, USA). Participants consisted of pairs of individuals with DSM-IV ${ }^{11}$ schizophrenia (schizophrenia group) and their unaffected siblings (schizophrenia-siblings group) ( $n=26$ pairs), and healthy controls (control group) and their siblings (controlsiblings group) ( $n=40$ pairs) (Table 1$)$. Siblings were full siblings, based on self-report. This cohort of individuals was substantially similar to that used in our previous reports on the thalamus and basal ganglia (122 participants in common $)^{14,15}$ and cingulate gyrus (128 participants in common). ${ }^{16}$ All participants gave written informed consent for participation following a complete description of the risks and benefits of the study.

Individuals in the schizophrenia group met DSM-IV criteria for schizophrenia and were all out-patients at the time of study. The control group had no lifetime history of any DSM-IV psychotic or major mood disorder (i.e. major depressive disorder and bipolar disorder) and no first-degree relatives with a psychotic disorder. Potential schizophrenia-siblings group participants were excluded if they had a lifetime history of any DSM-IV psychotic disorder, but not other DSM-IV Axis I disorders. Individuals in the control-siblings group met the same inclusion/exclusion criteria as the schizophrenia-siblings group (other than the requirement of having a sibling with schizophrenia) and were included to control for possible effects arising from the impact of psychiatric disorders other than schizophrenia. The schizophrenia-siblings group and control-siblings group had similar lifetime histories of DSM-IV defined substance dependence ( $23 \%$ and $8 \%$ respectively, $P=0.1$, Fisher's exact test), mood disorder $(50 \%$ and $35 \%, P=0.3)$ and anxiety disorder $(23 \%$ and $15 \%, P=0.5)$. The schizophrenia group were treated with antipsychotic medications (primarily atypicals), and had been clinically stable for at least 2 weeks prior to their participation. People in any group were excluded if they met DSM-IV criteria for substance abuse or dependence within the 3 months preceding assessment. Further details of participant recruitment, assessment, antipsychotic medication and lifetime comorbidities are available elsewhere. ${ }^{14,15}$ 


\begin{tabular}{|c|c|c|c|c|c|}
\hline Characteristics & $\begin{array}{l}\mathrm{SCZ}^{\mathrm{b}} \\
n=26\end{array}$ & $\begin{array}{l}\text { SCZ-SIB } \\
n=26\end{array}$ & $\begin{array}{l}\text { CON-SIB } \\
n=40\end{array}$ & $\begin{array}{l}\text { CON } \\
n=40\end{array}$ & $P^{\mathrm{C}}$ \\
\hline Age, years: ${ }^{d}$ mean (s.d.) & $22.7(3.0)$ & $22.3(3.4)$ & $20.3(3.6)$ & $21.2(3.6)$ & 0.02 \\
\hline $\begin{array}{l}\text { Gender, } n(\%) \\
\text { Female } \\
\text { Male }\end{array}$ & $\begin{array}{r}4(15.4) \\
22(84.6) \\
\end{array}$ & $\begin{array}{l}16(61.5) \\
10(38.5)\end{array}$ & $\begin{array}{l}28(70.0) \\
12(30.0)\end{array}$ & $\begin{array}{l}17(42.5) \\
23(57.5)\end{array}$ & $<0.001$ \\
\hline $\begin{array}{l}\text { Ethnicity, } n(\%) \\
\text { African American } \\
\text { White }\end{array}$ & $\begin{array}{r}9(34.6) \\
17(65.4)\end{array}$ & $\begin{array}{r}9(34.6) \\
17(65.4)\end{array}$ & $\begin{array}{r}8(20.0) \\
32(80.0)\end{array}$ & $\begin{array}{r}8(20.0) \\
32(80.0)\end{array}$ & 0.25 \\
\hline $\begin{array}{l}\text { Handedness, }{ }^{e} n(\%) \\
\text { Right } \\
\text { Left }\end{array}$ & $\begin{array}{r}22(84.6) \\
4(15.4)\end{array}$ & $\begin{array}{r}23(88.5) \\
3(11.5)\end{array}$ & $\begin{array}{c}37(92.5) \\
3(7.5)\end{array}$ & $\begin{array}{r}34(85.0) \\
6(15.0)\end{array}$ & 0.75 \\
\hline Parental SES, ${ }^{f}$ mean (s.d.) & $3.1(1.2)$ & $3.0(1.1)$ & $3.1(0.9)$ & $2.9(0.9)$ & 0.82 \\
\hline $\begin{array}{l}\text { Crystallised IQ, }{ }^{g} \text { least square mean (s.e) [\% difference } \\
\text { relative to the SCZ-SIB value] }\end{array}$ & $9.0(0.81)[-14.3]$ & $10.5(0.65)$ & $11.9(0.53)$ [13.3] & $11.7(0.49)$ [11.4] & 0.03 \\
\hline $\begin{array}{l}\text { Non-PFC cortical GM volume, }{ }^{\mathrm{h}} \mathrm{cm}^{3} \text { : least square mean } \\
\text { (s.e) [\% difference relative to the SCZ-SIB value] }\end{array}$ & $349(8.9)[-3.6]$ & $362(5.9)$ & $390(6.0)$ [7.7] & 381 (6.3) [5.3] & 0.006 \\
\hline $\begin{array}{l}\text { Non-PFC white matter surface area, }{ }^{i} \mathrm{~cm}^{2} \text { : least square } \\
\text { mean (s.e) [\% difference relative to the SCZ-SIB value] }\end{array}$ & 1348 (31.7) [-3.7] & $1399(24.8)$ & 1485 (22.3) [6.2] & 1464 (19.9) [4.7] & 0.02 \\
\hline $\begin{array}{l}\text { Mean cortical thickness, }{ }^{j} \mathrm{~mm} \text { : least square mean (s.e) } \\
\text { [\% difference relative to the SCZ-SIB value] }\end{array}$ & $2.43(0.016)[-0.8]$ & $2.45(0.013)$ & $2.49(0.013)$ [1.6] & $2.47(0.013)[0.8]$ & 0.04 \\
\hline \multicolumn{6}{|c|}{ 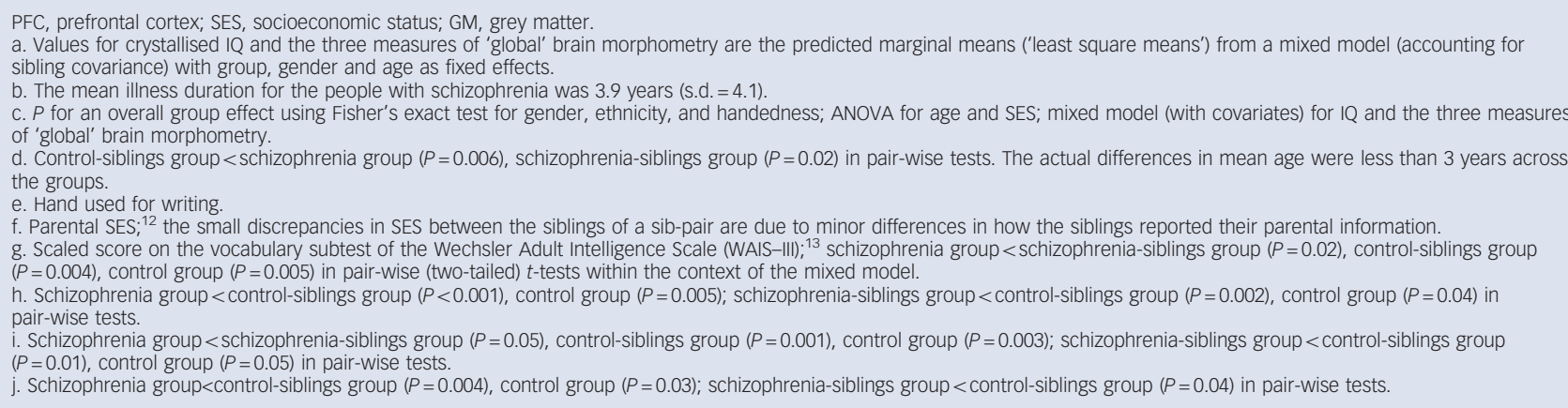 } \\
\hline
\end{tabular}

\section{Clinical and cognitive assessments}

Psychopathology and cognitive function were assessed as previously described ${ }^{14,17}$ using a battery of clinical and neuropsychological tests that were $z$-scored (against a centre-wide sample) and subsequently averaged within domains to yield summary scores on three domains of clinical symptoms - positive symptoms, negative symptoms, and thought disorganisation and four broad cognitive domains - working memory, episodic memory, executive function and attention. The mean scores of these domains by participant group were nearly identical to those reported previously. ${ }^{14}$

\section{Image collection and preparation}

Magnetic resonance scans were collected using a Siemens Magnetom Vision 1.5 T imaging system using a standard head coil. The magnetic resonance scanning protocol included the collection of multiple (2-4) high-resolution, three-dimensional $T_{1}$-weighted MPRAGE volumes: voxel size $1 \times 1 \times 1.25 \mathrm{~mm}^{3}$, repetition time (TR) $1765 \mathrm{~ms}$, inversion time (TI) $640 \mathrm{~ms}^{3}$, echo time (TE) $4.0 \mathrm{~ms}$, flip angle $10^{\circ}$, and scan time $6.5 \mathrm{~min}$ per acquisition. All MPRAGE scans for each individual were aligned and averaged to create a low-noise image volume, which was then tri-linearly interpolated into $0.5 \times 0.5 \times 0.5 \mathrm{~mm}^{3}$ isotropic voxels to produce smoother intensity histograms for more accurate segmentation. A region of interest containing frontal cortex was defined in the preprocessed image volume, and non-brain tissue (e.g. skull and dura) manually removed. Gaussian mixture modelling was used to segment this region into grey matter, white matter and cerebrospinal fluid, typically using three Gaussians to model each tissue class. The validity of this approach to tissue segmentation of prefrontal cortex has been previously established. ${ }^{18}$ A topologically correct surface (i.e. no 'holes' or 'handles' in the surface mesh) was then generated at the white/grey matter interface. ${ }^{19}$ References to simply 'surface' or 'surface area' refer to the white/grey matter interface.

\section{Delineation of prefrontal subregions}

Four raters, masked to the group status of the participants, used dynamic programming ${ }^{20}$ to manually delineate the sulcal boundaries of the superior, middle and inferior frontal gyri on the frontal white matter surface using a set of detailed anatomical rules $^{21}$ (Fig. 1, see online Fig. DS1 for a colour version of this figure). There was a high degree of both inter- and intrarater consistency in the delineation of these boundaries, with the average surface overlap exceeding $94 \%$ for both inter- and intrarater comparisons (see online supplement for details).

\section{Prefrontal measures}

The volume and thickness of each prefrontal subregion was calculated from labelled cortical distance maps. ${ }^{25,26}$ Grey matter volume was determined as the volume represented by the grey matter-labelled voxels in the tissue-segmented volume for which 


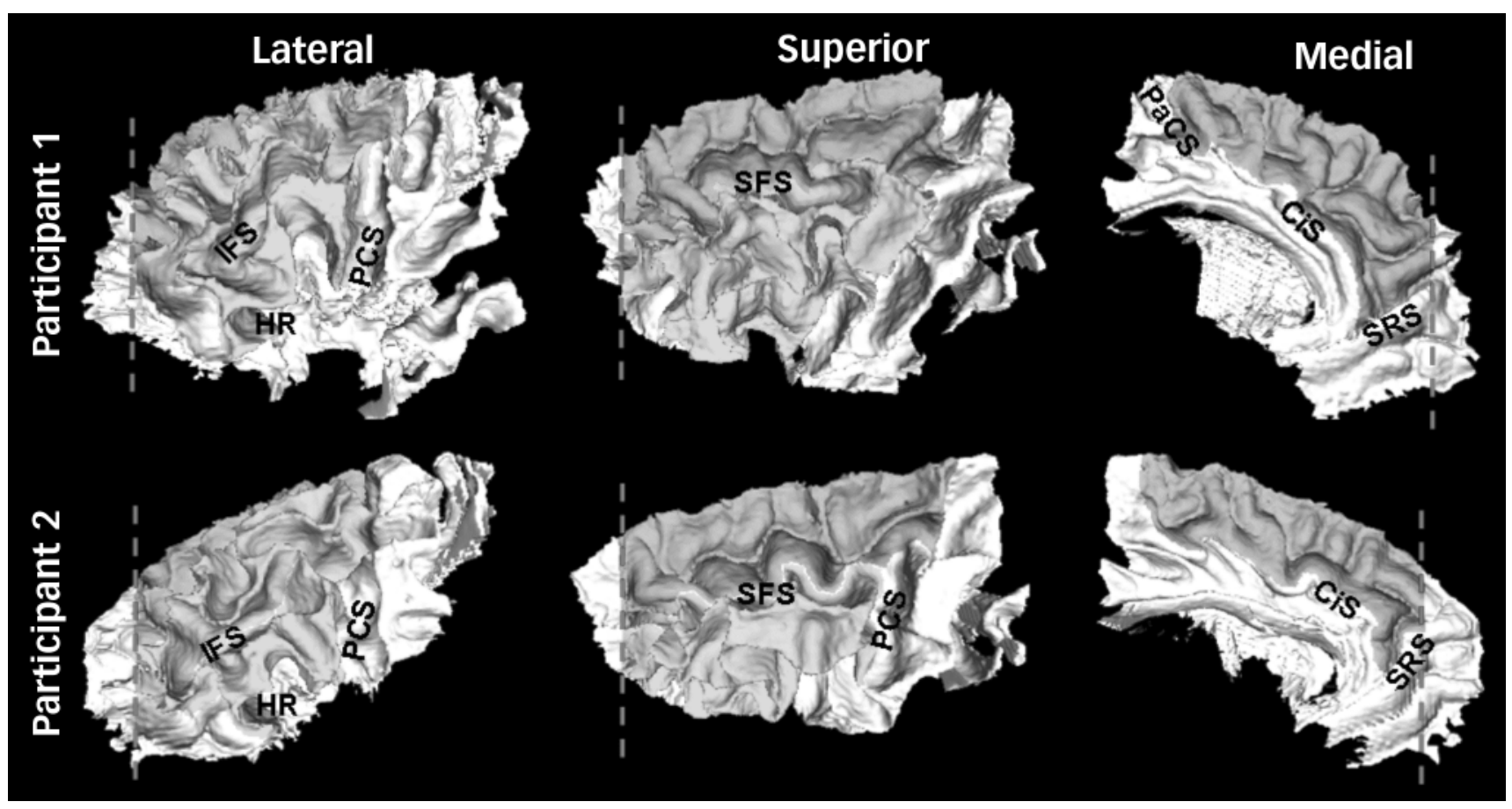

Fig. 1 Examples of the parcellated white matter surface of the prefrontal cortex in two participants.

In general, the boundaries for the superior, middle and inferior frontal gyri followed the depth of prominent sulci, except when necessary to cross over the crown of a gyrus to bridge an interrupted sulcus. The posterio-lateral border for all three regions was the precentral sulcus (PCS), using the most posterior sulcus in cases of a forked termination or parallel sulci. The posterio-medial limit of the superior frontal gyrus was the paracentral sulcus (PaCS), which typically is an extension of the superior precentral sulcus and/or a branch of the cingulate sulcus. The inferio-medial border of the superior frontal gyrus was the cingulate sulcus (CiS), so that the more superior paracingulate sulcus was considered part of the superior frontal gyrus if present. ${ }^{22}$ The anterio-inferio-medial border of the superior frontal gyrus was the superior rostral sulcus (SRS) whose posterior aspect was extended to intersect the cingulate sulcus if necessary to complete the inferio-medial boundary of the superior frontal gyrus. Anterio-laterally, there is no consistent sulcal boundary to delimit prefronta cortex from the cytoarchitectonically distinct frontal pole. Therefore, a frontal pole plane (dashed line) was defined as the coronal section (in an anterior/posterior-commissure oriented brain) passing through the anterior termination of the olfactory sulcus. ${ }^{21}$ This frontal pole plane defined the anterior limit of the superior and middle frontal gyrus (regardles of any naturally occurring sulci that infrequently might be present). Frequently the superior rostral sulcus does not extend to the frontal pole plane and in these cases the anteriomedial boundary of the superior frontal gyrus was completed by connecting the frontal pole plane to the superior rostral sulcus (along the superior edge where the surface transitions from the medial bank to the lateral convexity). Laterally, the superior frontal sulcus (SFS) and inferior frontal sulcus (IFS) defined the boundary between the superior/middle frontal gyri and middle/inferior frontal gyri respectively. The superior frontal sulcus nearly always intersects the frontal pole plane, but the inferior frontal sulcus typically had to be extended to the frontal pole plane to complete the anterio-inferior border of the middle frontal gyrus. The inferior and anterior border of the inferior frontal gyrus, moving from posterior to anterior, progressed from (a) the circular sulcus in the operculum to (b) the sulcus immediately ventral to the horizontal ramus (HR) of the lateral fissure to (c) the most prominent dorsally-progressing sulcus immediately anterior to the anterior termination of the horizontal ramus to (d) the inferior frontal sulcus. Connections between these sulci were bridged when necessary. Using the nomenclature of other authors, the sulci from stages (b)-(c) of this progression would represent the lateral frontomarginal and/or radiate sulcus of Petrides \& Pandy $a^{23}$ or one of the variants of the fronto-orbital sulcus of Ono et al. ${ }^{24}$ By this definition, the inferior frontal gyrus included a small portion of pars orbitalis immediately ventral to the horizontal ramus, but consisted primarily of pars opercularis and pars triangularis, similar to Buchanan et al. ${ }^{3}$ (see online Fig. DS1 for a colour version of this figure.)

the closest vertex on the overall frontal white matter surface was part of the delineated subsurface of interest (i.e. superior, middle or inferior frontal gyrus). The distance between each grey matter voxel and its associated subsurface was also computed to yield a thickness histogram, and the ninetieth percentile was chosen to represent cortical thickness. The area of each delineated subsurface was calculated from the triangulated graph of the white matter surface.

\section{Anatomical covariates}

To control for 'global' brain differences between groups, covariates were derived using FreeSurfer (version 3.0.4; http://surfer.nmr.mgh. harvard.edu), a software package for segmentation and cortical surface reconstruction. ${ }^{27,28}$ Separate covariates were derived for volume, area and thickness analyses to match the variable being analysed. Specifically, covariates for volume and area analyses were defined as FreeSurfer's estimate of total cortical grey matter volume or white matter surface area (summed across left and right hemispheres) respectively, minus total labelled cortical distance maps-derived volume or area summed across the superior, middle and inferior frontal gyri. This subtraction was done because the superior, middle and inferior frontal gyrus regions together constitute an appreciable portion of the total brain and thus including those regions in a covariate for brain size could remove variance specifically associated with the prefrontal cortex itself. An analogous calculation was not possible for thickness (since thickness is an 'average' rather than a summed measure); therefore, the covariate for thickness analyses was the FreeSurfer estimate of average thickness across the entire cortical grey matter. Thus, our analyses of thickness in the prefrontal cortex may be biased somewhat towards a negative result since the prefrontal cortex itself was included in the computation of the thickness covariate.

\section{Statistical analysis}

Group differences in volume, thickness, and area of the prefrontal cortex were assessed using mixed-models that estimated the covariance (correlation) in the residuals due to the sibling relationships as well as hemisphere (using PROC MIXED of SAS 9.1, SAS Institute Inc, Cary, North Carolina, USA). Group, hemisphere, group $\times$ hemisphere, gender and age were fixed-effect predictors, and a covariate for 'global' brain variation was also included (matched to the corresponding type of dependent variable; see above). IQ was not included as a covariate, since decreased cognitive performance is itself a feature of schizophrenia. Results for area and thickness are presented for each gyral region 
regardless of whether the volume differed across groups, since area and thickness have unique genetic influences. ${ }^{29}$ However, to partially control for multiple comparisons, we required the main effect of group to satisfy $P \leqslant 0.01$ for any of the three morphometric measures to be considered significant, after which pair-wise comparisons (two-tailed) between groups were examined. For the measures with a main effect of group, correlations with the psychopathology and neurocognitive scores were assessed in the schizophrenia group and schizophrenia-siblings group (separately) using non-parametric Spearman's correlations, partialling out 'global' brain variation, with $P \leqslant 0.01$ again used as the threshold to partially control for multiple correlations.

\section{Results}

\section{Prefrontal cortex volume}

There was a significant effect of group on grey matter volume of the middle and inferior, but not the superior frontal gyrus. The group effect for middle frontal gyrus volume was because of a reduced volume in the schizophrenia group (10-11\%, Table 2 and Fig. 2) relative to the schizophrenia-siblings group and both the control groups (all $P<0.02$ ). In contrast, the group effect for inferior frontal gyrus volume was a result of reduced volume in both the schizophrenia group and schizophrenia-siblings group, with the volume of the latter falling intermediate between the schizophrenia group and the two control groups $-11 \%$ greater than the schizophrenia group $(P=0.06)$, but $7-10 \%$ less than the control-siblings group and the control group $(P=0.06$ in a contrast of the schizophrenia-siblings group against the average of the two control groups). There was no effect of hemisphere $(P>0.24)$, or group $\times$ hemisphere interaction $(P>0.10)$ on volume of any of the three prefrontal cortex regions.

We conducted a males-only analysis to examine whether the results for inferior and middle frontal gyrus volume might have been confounded because of gender differences across groups. The results of this analysis were consistent with the full cohort of participants; i.e. the main effect of group on grey matter volume remained significant in inferior frontal gyrus
$(F(3,24)=5.7, \quad P=0.004)$ and trended towards significant in middle frontal gyrus $(F(3,33)=2.5, P=0.08$; see online supplement $)$.

\section{Prefrontal cortex area and thickness}

The effect of group was not significant for either surface area or cortical thickness of any of the three regions. There was no group $\times$ hemisphere interaction for either measure for any of the three prefrontal cortex regions $(P=0.07$ for area of superior frontal gyrus, otherwise $P>0.23$, and no main effect of hemisphere on area of any region $(P>0.3)$. There was a strong main effect of hemisphere on thickness in all three regions $(F>44$, $P<0.0001$ ), with the direction of this asymmetry differing across the regions (left $>$ right in superior frontal gyrus, right $>$ left in middle and inferior frontal gyri).

Examination of the correlation between thickness and area, computed across all participants, indicated that these two measures yielded largely independent information regarding prefrontal morphometry (Spearman's $r<0.2$, for both hemispheres in all three regions). Nonetheless, at the level of the group averages, the patterns of both area and thickness values across groups were qualitatively similar to the volume differences across groups within the middle and inferior frontal gyri (i.e. smallest in the schizophrenia group; intermediate in the schizophreniasiblings group for inferior frontal gyrus). However, only the volume measure, which is conceptually the product of area and thickness, detected statistically significant group differences in this study.

\section{Covariates}

The main effect of group on middle and inferior frontal gyrus volume remained significant when dichotomous covariates for lifetime history of substance dependence, mood disorders and anxiety disorders were included in the mixed model (middle frontal gyrus: $F(3,67)=4.1, P=0.01$; inferior frontal gyrus: $F(3,64)=3.9, P=0.01)$. Furthermore, each of these covariates themselves had a non-significant influence on middle and inferior frontal gyrus volume ( $P>0.1$ for all). These results, along with the similar aggregate lifetime histories of the schizophrenia-siblings

\begin{tabular}{|c|c|c|c|c|c|c|}
\hline Region & $\begin{array}{l}\text { SCZ, least square mean } \\
\text { (s.e) [\% difference relative } \\
\text { to the SCZ-SIB value] }\end{array}$ & $\begin{array}{l}\text { SCZ-SIB, } \\
\text { least square } \\
\text { mean (s.e) }\end{array}$ & $\begin{array}{l}\text { CON-SIB, least square mean } \\
\text { (s.e) [\% difference relative } \\
\text { to the SCZ-SIB value] }\end{array}$ & $\begin{array}{l}\text { CON, least square mean } \\
\text { (s.e) [\% difference relative } \\
\text { to the SCZ-SIB value] }\end{array}$ & $F$ & $P$ \\
\hline \multicolumn{7}{|c|}{ Superior frontal gyrus } \\
\hline Volume & $24.6(0.86)[-7.5]$ & $26.6(0.92)$ & $26.5(0.66)[-0.4]$ & $27.1(0.58)[1.9]$ & 2.0 & 0.13 \\
\hline Area & $61.9(1.98)[-2.8]$ & $63.7(2.04)$ & $61.3(1.50)[-3.8]$ & $63.5(1.41)[-0.3]$ & 0.6 & 0.64 \\
\hline Thickness & $3.58(0.054)[-4.5]$ & $3.75(0.060)$ & $3.77(0.054)[0.5]$ & $3.77(0.051)$ [0.5] & 2.5 & 0.06 \\
\hline \multicolumn{7}{|c|}{ Middle frontal gyrus } \\
\hline Volume $^{b}$ & $18.6(0.56)[-11.4]$ & $21.0(0.56)$ & $20.7(0.58)$ [-1.4] & $20.7(0.53)[-1.4]$ & 3.8 & 0.01 \\
\hline Area & $51.5(1.17)[-6.9]$ & $55.3(1.24)$ & $53.3(1.24)[-3.6]$ & $53.5(1.15)[-3.3]$ & 1.8 & 0.16 \\
\hline Thickness & $3.18(0.052)[-3.6]$ & $3.30(0.047)$ & $3.29(0.046)[-0.3]$ & $3.29(0.041)[-0.3]$ & 1.2 & 0.32 \\
\hline \multicolumn{7}{|c|}{ Inferior frontal gyrus } \\
\hline Volume $e^{c}$ & $11.2(0.51)$ [-9.7] & $12.4(0.52)$ & $13.8(0.38)$ [11.3] & $13.3(0.37)[7.3]$ & 4.7 & 0.005 \\
\hline Area & $29.8(1.08)[-4.8]$ & $31.3(1.04)$ & $33.2(0.78)[6.1]$ & $32.3(0.77)[3.2]$ & 1.9 & 0.14 \\
\hline Thickness & 3.39 (0.073) [-4.0] & $3.53(0.064)$ & $3.63(0.060)[2.8]$ & $3.62(0.060)$ [2.5] & 2.3 & 0.08 \\
\hline \multicolumn{7}{|c|}{$\begin{array}{l}\text { SCZ, schizophrenia group; SCZ-SIB, schizophrenia-siblings group; CON, control group; CON-SIB, control-siblings group. } \\
\text { a. Values are least square means by participant group for superior, middle and inferior frontal gyrus volume }\left(\mathrm{cm}^{3}\right) \text {, area }\left(\mathrm{cm}^{2}\right) \text { and thickness (mm) from a mixed model with group, } \\
\text { hemisphere, group } \times \text { hemisphere, age, gender and corresponding 'global' brain covariate (non-prefrontal cortical grey matter volume, non-prefrontal white matter surface area and } \\
\text { mean cortical thickness for volume, area and thickness respectively) as fixed effects. As the least square means collapse across hemisphere, the values for volume and area should } \\
\text { be interpreted as those for a single representative 'average' hemisphere (i.e. the volume and area values need to be doubled for an approximation of the whole brain total). } F \text { and } P \\
\text { are for the main effect of group (using type III tests). The degrees of freedom (d.f.) for the } F \text { statistic were } 3 \text { for the numerator, and ranged between } 56 \text { and } 67 \text { for the denominator } \\
\text { (even though all participants contributed data for all gyri and measures) because d.f.'s were calculated using the method of Kenward } \& \text { Roger. } \\
\text { b. Schizophrenia group }<\text { schizophrenia-siblings group }(P=0.004) \text {, control-siblings group }(P=0.02) \text {, control group }(P=0.008) \text { in pair-wise (two-tailed) } t \text {-tests within the context of the } \\
\text { mixed model. } \\
\text { c. Schizophrenia group }<\text { schizophrenia-siblings group (trend, } P=0.06) \text {, control-siblings group }(P<0.001) \text {, control group }(P=0.002) \text {; schizophrenia-siblings group }<\text { control-siblings group } \\
(P=0.04) \text { in pair-wise tests. }\end{array}$} \\
\hline
\end{tabular}



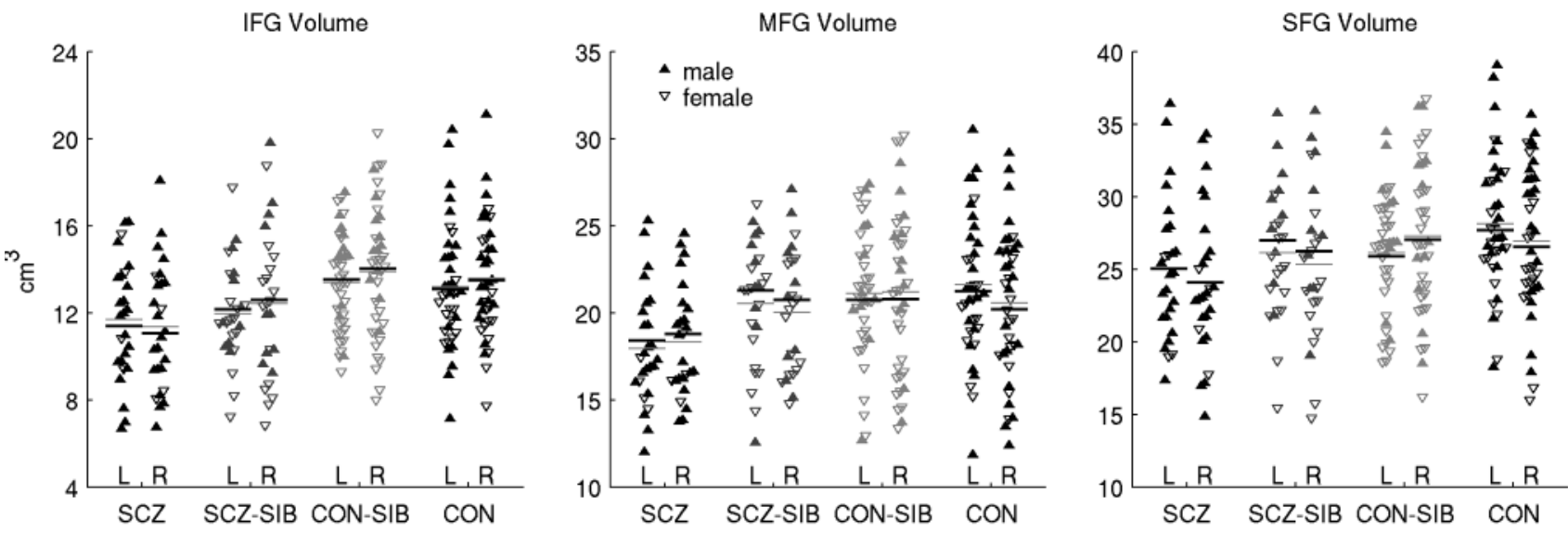

Fig. 2 Plots of raw inferior (IFG), middle (MFG) and superior frontal gyri (SFG) volumes separated by participant group and hemisphere.

Filled triangles are males; open triangles are females. Black horizontal lines indicate the predicted marginal means ('least square means') from the full statistical model, which included gender, age and non-prefrontal cortical grey matter volume as covariates. Thin grey lines indicate the mean of the raw values. L, left: R, right; SCZ, schizophrenia group; SCZ-SIB, schizophrenia-siblings group; CON, control group; CON-SIB, control-siblings group.

group and control-siblings group on these variables, suggest that the inferior frontal gyrus deficit in the schizophrenia-siblings group cannot be simply attributed to the presence of nonpsychotic Axis I disorders.

The groups differed on the three measures used as covariates to control for overall brain changes (at $P<0.05$, rather than $P<0.01$, for two of the measures; Table 1). Mixed model analyses of the prefrontal cortex measures without their associated brain covariate indicated at least trend-level evidence for group differences $(P<0.1$ for main effect of group) for all measures and regions except for area of the superior frontal gyrus, indicating that superior, middle and inferior frontal gyrus morphometry was broadly altered in these individuals, even if a portion of these differences reflected changes proportional to overall brain differences rather than regional changes.

\section{Relationships of structural measures with psychopathology and neurocognition}

The correlation of middle and inferior frontal gyrus volume (summed across left and right hemispheres) with duration of illness, crystallised IQ, the three domain scores of psychopathology and four neurocognition scores was separately assessed in the schizophrenia group and schizophrenia-siblings group. Correlations were controlled for non-prefrontal cortical grey matter volume. Duration of illness in the schizophrenia group was not correlated with volume of the middle or inferior frontal gyri $(P>0.2)$. The only significant correlations $(P \leqslant 0.01)$ were between middle frontal gyrus volume and disorganised symptoms in the schizophrenia-siblings group (Spearman's $r=-0.51$, $P=0.01$ ) and between inferior frontal gyrus volume and negative symptoms in the schizophrenia group $(r=-0.58, P=0.003)$.

\section{Discussion}

Our results are consistent with regional abnormalities in the structure of the prefrontal cortex of individuals with schizophrenia and their siblings. Grey matter volume was reduced in both the middle and inferior frontal gyri in individuals with schizophrenia, relative to those in both control groups. However, only in the inferior frontal gyrus was the grey matter volume in the schizophrenia-siblings group also reduced, resulting in a volume intermediate between the schizophrenia group and both control groups. These group effects were demonstrated while controlling for 'global' brain changes in non-prefrontal cortical grey matter volume, which itself differed between groups.

\section{Structural phenotypes for schizophrenia risk}

Of the structural measures investigated in this study, grey matter volume of the inferior frontal gyrus was most clearly related to familial risk for schizophrenia. Additive genetic factors represent a likely source for the presence of inferior frontal gyrus deficits in both the affected and unaffected siblings, thus implicating inferior frontal gyrus volume as a potential endophenotype for schizophrenia. ${ }^{31}$ Although shared environmental factors (e.g. similar intrauterine factors) cannot be excluded as a source of these inferior frontal gyrus deficits, twin and adoption studies indicate that shared environmental factors have a negligible contribution to the manifestation of schizophrenia itself. ${ }^{32,33}$ Middle frontal gyrus volume deficits were only observed in the schizophrenia group, which suggests that changes in middle frontal gyrus structure are related to the presence of disease rather than familial risk.

We are not aware of other studies that employed manual frontal parcellation (of either a magnetic resonance imaging volume or a sulcal/gyral surface model) to investigate the localisation of prefrontal cortical changes in the relatives of individuals with schizophrenia. However, regional brain morphometry in such unaffected family members has been investigated using automated whole-brain analysis tools. In one large cohort of individuals, assessed using both voxel-based morphometry and FreeSurfer, there was no evidence for decreased grey matter volume or thickness in the lateral frontal gyri of siblings without psychosis compared with healthy controls, even at exploratory thresholds. ${ }^{34,35}$ Similar null results in the lateral prefrontal cortex of relatives have been reported by others. ${ }^{36-38}$ However, still other family studies have reported associations between genetic liability to schizophrenia and reduced grey matter in middle and inferior frontal gyri. ${ }^{39-41}$ Our results support a relationship between genetic risk and grey matter volume in the inferior, but not middle frontal gyrus. The discrepant results across studies could be a result of either variation in analysis methods or true cohort differences. 


\section{Prefrontal structural deficits in individuals with schizophrenia}

At least six previous in vivo neuroimaging studies of individuals with schizophrenia have partitioned the prefrontal cortex into the superior, middle and inferior frontal gyri. ${ }^{2-7}$ Four of these six studies found evidence for deficits in inferior frontal gyrus volume in the individuals with schizophrenia, whereas two found evidence for middle frontal gyrus deficits, and only one found evidence for superior frontal gyrus deficits. Notably, voxel-based morphometry studies also report deficits more frequently in inferior than middle frontal gyri in individuals with schizophrenia compared with controls. ${ }^{42}$ Other automated whole-brain analyses provide further support for the existence of deficits in the inferior frontal gyrus, with comparatively spared middle frontal gyrus. ${ }^{43-45}$ This suggests that grey matter reduction in lateral prefrontal cortex in schizophrenia is most prominent in the inferior frontal gyrus, and progressively less so in the middle and superior frontal gyri, a conclusion consistent with the present study.

\section{Relationships with psychopathology and neurocognition}

The observed correlation between smaller inferior frontal gyrus volumes and increased negative symptoms in the schizophrenia group is consistent with literature linking inferior frontal cortex to language function, motivation and goal-directed action generation. ${ }^{46-49}$ Although the siblings of individuals with schizophrenia also exhibit increased negative symptoms, ${ }^{17}$ a correlation between inferior frontal gyrus volume and negative symptoms was not evident in the schizophrenia-siblings group, suggesting that disease-specific factors could be driving this association in the affected siblings. Given the role of subregions of the middle frontal gyrus (i.e. Brodmann's area 46) in executive control and working memory, we had also hypothesised, but did not find, a correlation of middle frontal gyrus volume with either of these cognitive domains in the schizophrenia group (see Zuffante et al for a similar result). ${ }^{50}$ Overall, more studies are necessary before firm conclusions can be drawn regarding the correlation (or lack thereof) of cognitive and clinical variables with structural measures at the level of sublobular (e.g. gyral) partitions.

\section{Limitations}

The sample size of this study was large enough to detect significant main effects of group for inferior and middle frontal gyrus volume (representing pair-wise group differences of 10-20\%), but likely too small to reliably detect (e.g. with $80 \%$ power) differences on the order of $5-10 \%$ between groups. ${ }^{51}$ Notably, however, there was not even trend-level evidence for either superior or middle frontal gyrus volume as an intermediate phenotype in the unaffected siblings since the volume of both gyri in the schizophrenia-siblings group was very similar to the controls. Formally testing the heritability of inferior frontal gyrus volume (i.e. 'familiality' in the context of our sibling study design $)^{52}$ could strengthen the case for inferior frontal gyrus volume as a potential endophenotype of genetic risk for schizophrenia. Also, the groups differed in their gender distribution, although gender was included as a covariate and a males-only analysis supported the results obtained using all participants.

The effects of antipsychotic medication on cortical tissue are a potential confound in interpreting studies of brain changes in schizophrenia. In one study involving monkeys, chronic exposure to either haloperidol or olanzapine reduced the weight of the frontal cortex compared with sham-treated monkeys. ${ }^{53}$ However, available in vivo imaging data in humans suggests that people with first-episode schizophrenia taking atypical antipsychotics are less likely to exhibit frontal grey matter deficits relative to controls than people on typical antipsychotics. ${ }^{54,55}$ It remains unknown whether this differential reflects a positive therapeutic effect of atypical antipsychotics or a possible neurotoxic effect of typical agents. Regardless, since atypical antipsychotics were the predominant medication of our schizophrenia group, the implication of these medication studies in humans is that the deficits observed in this group were if anything attenuated relative to a hypothetical sample of similarly aged but non-medicated individuals. Furthermore, our finding of decreased inferior frontal gyrus volume in the unaffected siblings cannot be attributed to medication effects since they had never been medicated.

In the current study, we partitioned prefrontal cortex by delineating gyral regions directly on a model of the white matter surface, which was helpful in visualising the variable folding patterns of prefrontal cortex. The manual nature of this gyral delineation was time consuming and might have introduced additional variability compared with more automated approaches. However, we demonstrated a high degree of both inter- and intrarater consistency in the delineation of the gyral boundaries, and studies using manual definition of regions of interest are still considered the 'gold-standard' by many investigators. Studies directly comparing the results of different analysis approaches on a common data-set are necessary in order to better understand the extent to which methodological differences may influence group comparisons.

Previously, we have reported on the structure of the thalamus, ${ }^{14}$ caudate, putamen and globus pallidus, ${ }^{15}$ and anterior and posterior cingulate gyrus ${ }^{16}$ in largely overlapping cohorts of individuals, using analysis approaches specifically designed and validated for these structures. None of these previously examined structures exhibited a statistically significant effect of group on regional volumes after controlling for brain volume. Although the multiple structures raise a potential multiple comparisons issue, we note that we used a more stringent threshold $(P \leqslant 0.01)$ for the group effect in the present study. Furthermore, the structures investigated in this study have already been implicated in other studies as potentially involved in the pathophysiology of schizophrenia, which partially ameliorates the multiple comparison problem. To further understand the regional specificity of our findings for inferior and middle frontal gyri, whole brain cortical mapping ${ }^{41,44}$ would permit localisation of the most prominent deficits across the whole cortex, and thus would be a useful complimentary analysis of the current cohort.

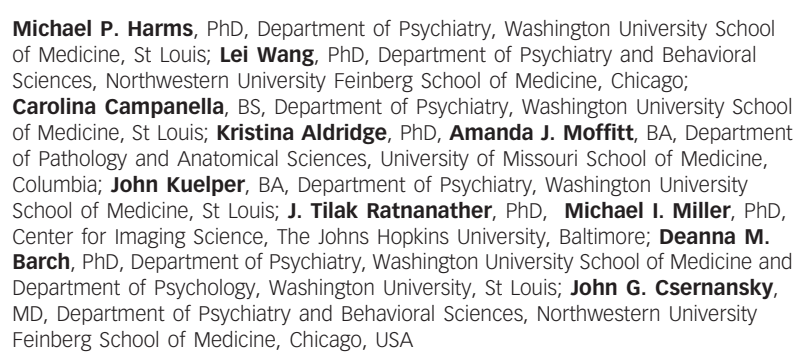

Michael P. Harms, PhD, Department of Psychiatry, Washington University School of Medicine, St Louis; Lei Wang, PhD, Department of Psychiatry and Behavioral Sciences, Northwestern University Feinberg School of Medicine, Chicago;

Carolina Campanella, BS, Department of Psychiatry, Washington University School of Medicine, St Louis; Kristina Aldridge, PhD, Amanda J. Moffitt, BA, Department of Pathology and Anatomical Sciences, University of Missouri School of Medicine, Columbia; John Kuelper, BA, Department of Psychiatry, Washington University School of Medicine, St Louis; J. Tilak Ratnanather, PhD, Michael I. Miller, PhD, Center for Imaging Science, The Johns Hopkins University, Baltimore; Deanna M. Barch, PhD, Department of Psychiatry, Washington University School of Medicine and Department of Psychology, Washington University St Louis; John G. Csernansky, DiD, Department of Psychiatry and Behavioral Sciences, Northwestern University Feinberg School of Medicine, Chicago, USA

Correspondence: Michael P. Harms, PhD, Department of Psychiatry (Box 8134), Washington University School of Medicine, 660 S. Euclid Ave, St Louis, MO 63110, USA. Email: mharms@conte.wustl.edu.

First received 16 Apr 2009, final revision 15 Jul 2009, accepted 21 Oct 2009

\section{Funding}

This work was supported by the National Institute of Mental Health (NIMH) (MH071616, MH056584) and the National Center for Research Resources (RR015241). 


\section{Acknowledgements}

We thank the staff of the Administration/Assessment Core of the CCNMD at Washington University School of Medicine for collection of the clinical and imaging data, and the Biostatistical Core, directed by Paul A. Thompson and Xuan Huang, for data management and statistical consultation.

\section{References}

1 Barch DM. The cognitive neuroscience of schizophrenia. In Annual Review of Clinical Psychology, Vol 1 (eds T Cannon, S Mineka): 321-53. American Psychological Association, 2005

2 Goldstein JM, Goodman JM, Seidman LJ, Kennedy DN, Makris N, Lee H, et al. Cortical abnormalities in schizophrenia identified by structural magnetic resonance imaging. Arch Gen Psychiatry 1999; 56: 537-47.

3 Buchanan RW, Vladar K, Barta PE, Pearlson GD. Structural evaluation of the prefrontal cortex in schizophrenia. Am J Psychiatry 1998; 155: 1049-55.

4 Yamasue $\mathrm{H}$, Iwanami A, Hirayasu $\mathrm{Y}$, Yamada $\mathrm{H}$, Abe $\mathrm{O}$, Kuroki $\mathrm{N}$, et al. ocalized volume reduction in prefrontal, temporolimbic, and paralimbic regions in schizophrenia: an MRI parcellation study. Psychiatry Res 2004 131: 195-207

5 Buchanan RW, Francis A, Arango C, Miller K, Lefkowitz DM, McMahon RP, et al. Morphometric assessment of the heteromodal association cortex in schizophrenia. Am J Psychiatry 2004; 161: 322-31.

6 Suzuki M, Zhou SY, Takahashi T, Hagino H, Kawasaki Y, Niu L, et al. Differential contributions of prefrontal and temporolimbic pathology to mechanisms of psychosis. Brain 2005; 128: 2109-22.

7 Crespo-Facorro B, Kim J, Andreasen NC, O'Leary DS, Magnotta V. Regional frontal abnormalities in schizophrenia: a quantitative gray matter volume and cortical surface size study. Biol Psychiatry 2000; 48: 110-9.

8 Lawrie SM, Whalley HC, Abukmeil SS, Kestelman JN, Donnelly L, Miller P, et al. Brain structure, genetic liability, and psychotic symptoms in subjects at high risk of developing schizophrenia. Biol Psychiatry 2001; 49: 811-23.

9 Cannon TD, van Erp TG, Huttunen M, Lonnqvist J, Salonen O, Valanne L, et al. Regional gray matter, white matter, and cerebrospinal fluid distributions in schizophrenic patients, their siblings, and controls. Arch Gen Psychiatry 1998; 55: 1084-91.

10 Staal WG, Hulshoff Pol HE, Schnack HG, Hoogendoorn ML, Jellema K, Kahn RS. Structural brain abnormalities in patients with schizophrenia and their healthy siblings. Am J Psychiatry 2000; 157: 416-21.

11 American Psychiatric Association. Diagnostic and Statistical Manual of Mental Disorders (4th edn) (DSM-IV). APA, 1994.

12 Hollingshead AB. Four factor index of social status. Yale University, 1975

13 Wechsler D. Wechsler Adult Intelligence Scale (3rd edn). Psychological Corporation, 1997.

14 Harms MP, Wang L, Mamah D, Barch DM, Thompson PA, Csernansky JG Thalamic shape abnormalities in individuals with schizophrenia and thei nonpsychotic siblings. J Neurosci 2007; 27: 13835-42.

15 Mamah D, Harms MP, Wang L, Barch D, Thompson P, Kim J, et al. Basal ganglia shape abnormalities in the unaffected siblings of schizophrenia patients. Biol Psychiatry 2008; 64: 111-20.

16 Calabrese DR, Wang L, Harms MP, Ratnanather JT, Barch DM, Cloninger CR, et al. Cingulate gyrus neuroanatomy in schizophrenia subjects and their nonpsychotic siblings. Schizophr Res 2008; 104: 61-70.

17 Delawalla Z, Barch DM, Fisher Eastep JL, Thomason ES, Hanewinkel MJ, Thompson PA, et al. Factors mediating cognitive deficits and psychopathology among siblings of individuals with schizophrenia. Schizoph Bull 2006; 32: 525-37.

18 Lee NA, Priebe CE, Miller MI, Ratnanather JT. Validation of alternating Kerne mixture method: application to tissue segmentation of cortical and subcortical structures. J Biomed Biotechnol 2008; 2008: 346129.

19 Han X, XU C, Braga-Neto U, Prince JL. Topology correction in brain cortex segmentation using a multiscale, graph-based algorithm. IEEE Trans Med Imaging 2002; 21: 109-21.

20 Khaneja N, Grenander U, Miller MI. Dynamic programming generation of curves on brain surfaces. IEEE Trans Pattern Anal Mach Intell 1998; 20: $1260-4$

21 John JP, Wang L, Moffitt AJ, Singh HK, Gado MH, Csernansky JG. Inter-rater reliability of manual segmentation of the superior, inferior and middle fronta gyri. Psychiatry Res 2006; 148: 151-63.

22 Fornito A, Wood SJ, Whittle S, Fuller J, Adamson C, Saling MM, et al. Variability of the paracingulate sulcus and morphometry of the medial frontal cortex: associations with cortical thickness, surface area, volume, and sulcal depth. Hum Brain Mapp 2008; 29: 222-36.
23 Petrides M, Pandya DN. The frontal cortex. In The Human Nervous System (eds G Paxinos, JK Mai). Elsevier Academic Press, 2004

24 Ono M, Kubik S, Abernathey CD. Atlas of the Cerebral Sulci: 92-93. Thieme Medical Publishers, 1990.

25 Miller MI, Hosakere M, Barker AR, Priebe CE, Lee N, Ratnanather JT, et al. Labeled cortical mantle distance maps of the cingulate quantify differences between dementia of the Alzheimer type and healthy aging. Proc Natl Acad SCi USA 2003; 100: 15172-7

26 Wang L, Hosakere M, Trein JC, Miller A, Ratnanather JT, Barch DM, et al. Abnormalities of cingulate gyrus neuroanatomy in schizophrenia. Schizophr Res 2007: 93: 66-78.

27 Dale AM, Fischl B, Sereno MI. Cortical surface-based analysis I. Segmentation and surface reconstruction. Neuroimage 1999; 9: 179-94.

28 Fischl B, Sereno MI, Dale AM. Cortical surface-based analysis. II. Inflation, flattening, and a surface-based coordinate system. Neuroimage 1999; 9: 195-207.

29 Panizzon MS, Fennema-Notestine C, Eyler LT, Jernigan TL, Prom-Wormley $E_{\text {, }}$ Neale $M$, et al. Distinct genetic influences on cortical surface area and cortical thickness. Cereb Cortex 2009; 19: 2728-35.

30 Kenward MG, Roger JH. Small sample inference for fixed effects from restricted maximum likelihood. Biometrics 1997; 53: 983-97.

31 Gottesman, II, Gould TD. The endophenotype concept in psychiatry: etymology and strategic intentions. Am J Psychiatry 2003; 160: 636-45.

32 Cannon TD, Kaprio J, Lonnqvist J, Huttunen M, Koskenvuo M. The genetic epidemiology of schizophrenia in a Finnish twin cohort. A population-based modeling study. Arch Gen Psychiatry 1998; 55: 67-74.

33 Cardno AG, Marshall EJ, Coid B, Macdonald AM, Ribchester TR, Davies NJ, et al. Heritability estimates for psychotic disorders: the Maudsley twin psychosis series. Arch Gen Psychiatry 1999; 56: 162-8.

34 Goldman AL, Pezawas L, Mattay VS, Fischl B, Verchinski BA, Chen Q, et al. Widespread reductions of cortical thickness in schizophrenia and spectrum disorders and evidence of heritability. Arch Gen Psychiatry 2009; 66: 467-77.

35 Honea RA, Meyer-Lindenberg A, Hobbs KB, Pezawas L, Mattay VS, Egan MF et al. Is gray matter volume an intermediate phenotype for schizophrenia? A voxel-based morphometry study of patients with schizophrenia and their healthy siblings. Biol Psychiatry 2008; 63: 465-74.

36 Job DE, Whalley HC, McConnell S, Glabus M, Johnstone EC, Lawrie SM. Voxel-based morphometry of grey matter densities in subjects at high risk of schizophrenia. Schizophr Res 2003; 64: 1-13.

37 Goghari VM, Rehm K, Carter CS, MacDonald 3rd AW. Regionally specific cortical thinning and gray matter abnormalities in the healthy relatives of schizophrenia patients. Cereb Cortex 2007; 17: 415-24.

38 Fan $\mathrm{Y}$, Gur RE, Gur RC, Wu X, Shen D, Calkins ME, et al. Unaffected family members and schizophrenia patients share brain structure patterns: a highdimensional pattern classification study. Biol Psychiatry 2008; 63: 118-24.

39 McIntosh AM, Job DE, Moorhead WJ, Harrison LK, Whalley HC, Johnstone EC, et al. Genetic liability to schizophrenia or bipolar disorder and its relationship to brain structure. Am J Med Genet B Neuropsychiatr Genet 2006; 141B: $76-83$.

40 McDonald C, Bullmore ET, Sham PC, Chitnis X, Wickham H, Bramon E, et al. Association of genetic risks for schizophrenia and bipolar disorder with specific and generic brain structural endophenotypes. Arch Gen Psychiatry 2004; 61: 974-84.

41 Cannon TD, Thompson PM, van Erp TG, Toga AW, Poutanen VP, Huttunen M, et al. Cortex mapping reveals regionally specific patterns of genetic and disease-specific gray-matter deficits in twins discordant for schizophrenia. Proc Natl Acad Sci USA 2002; 99: 3228-33.

42 Honea R, Crow TJ, Passingham D, Mackay CE. Regional deficits in brain volume in schizophrenia: a meta-analysis of voxel-based morphometry studies. Am J Psychiatry 2005; 162: 2233-45.

43 Davatzikos C, Shen D, Gur RC, Wu X, Liu D, Fan Y, et al. Whole-brain morphometric study of schizophrenia revealing a spatially complex set of focal abnormalities. Arch Gen Psychiatry 2005; 62: 1218-27.

44 Kuperberg GR, Broome MR, McGuire PK, David AS, Eddy M, Ozawa F, et al. Regionally localized thinning of the cerebral cortex in schizophrenia. Arch Gen Psychiatry 2003; 60: 878-88.

45 Venkatasubramanian G, Jayakumar PN, Gangadhar BN, Keshavan MS. Automated MRI parcellation study of regional volume and thickness of prefrontal cortex (PFC) in antipsychotic-naive schizophrenia. Acta Psychiatr Scand 2008; 117: 420-31.

46 Hagoort P. On Broca, brain, and binding: a new framework. Trends Cogn Sci 2005; 9: 416-23.

47 Bookheimer S. Functional MRI of language: new approaches to understanding the cortical organization of semantic processing. Ann Rev Neurosci 2002; 25: 151-88. 
48 Cheng $Y$, Meltzoff AN, Decety J. Motivation modulates the activity of the human mirror-neuron system. Cereb Cortex 2007; 17: 1979-86.

49 Hamilton AF, Grafton ST. Action outcomes are represented in human inferior frontoparietal cortex. Cereb Cortex 2008; 18: 1160-8.

50 Zuffante P, Leonard CM, Kuldau JM, Bauer RM, Doty EG, Bilder RM. Working memory deficits in schizophrenia are not necessarily specific or associated with MRI-based estimates of area 46 volumes. Psychiatry Res 2001; 108: 187-209.

51 Steen RG, Hamer RM, Lieberman JA. Measuring brain volume by MR imaging: impact of measurement precision and natural variation on sample size requirements. Am J Neuroradiol 2007; 28: 1119-25.

52 Kendler KS, Neale MC. 'Familiality' or heritability. Arch Gen Psychiatry 2009; 66: $452-3$.
53 Dorph-Petersen KA, Pierri JN, Perel JM, Sun Z, Sampson AR, Lewis DA The influence of chronic exposure to antipsychotic medications on brain size before and after tissue fixation: a comparison of haloperidol and olanzapine in macaque monkeys. Neuropsychopharmacology 2005; 30 1649-61.

54 Dazzan P, Morgan KD, Orr K, Hutchinson G, Chitnis X, Suckling J, et al. Different effects of typical and atypical antipsychotics on grey matter in first episode psychosis: the AESOP study. Neuropsychopharmacology 2005; $\mathbf{3 0}$ $765-74$

55 Lieberman JA, Tollefson GD, Charles C, Zipursky R, Sharma T, Kahn RS, et al. Antipsychotic drug effects on brain morphology in first-episode psychosis. Arch Gen Psychiatry 2005; 62: 361-70.

\section{psychiatry in pictures}

\section{Fossil Fools (2008), dry point etching}

Lee Galpin (b. 1958)

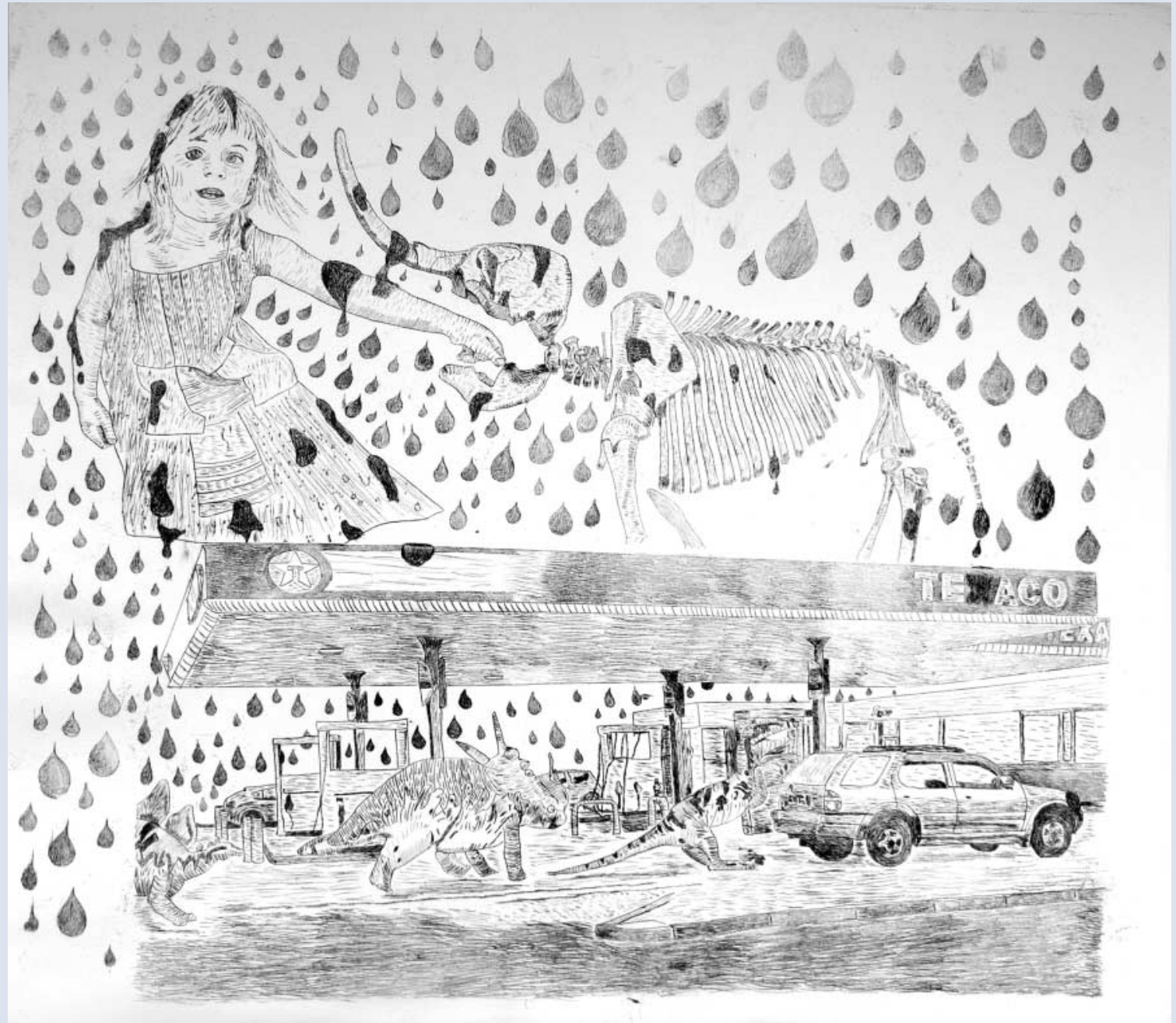

My work involves taking photos out of their intended context and creating a new world and life for them via a process called dry point etching. This image was created using a photo of a child from a fashion shoot, dinosaurs from a book and a photo I took of a local petrol station. The juxtaposition of all these disparate images aims to deliver a message about issues that concern us all and particularly our children. I found my artistic voice while attending occupational therapy at the Bethlem 3 years ago. The first time I saw my simple scratchings printed onto paper truly was a life-changing moment. I had always concentrated on work in a way that was damaging to my health. I now have an aim to my day, not just to work as many hours as I can, but to create something of which I can be proud, something that serves my spirit and my mind. 